among FSWs derived from independent data sources, was more consistent with observed HIV trends, and if these trends could have occurred without post-Avahan increases in CCU (two null hypotheses were assumed-one being more ( $\mathrm{HOb})$ and less conservative $(\mathrm{HOa})$ ). The most likely $\mathrm{CCU}$ hypothesis was used to predict the intervention impact on HIV prevalence/incidence and HIV infections prevented.

Results Using the most likely CCU hypothesis for each district (H1), results so far suggest that the increase in condom use postAvahan may have resulted in between 21 and 45\% of new HIV infections being averted among FSWs in Mysore, Belgaum and Bellary respectively from 2004 to 2007. Similar results were obtained for clients but the absolute number averted was 2-8 fold more. Model projections (Abstract S7.3 figure 1) suggest that this has resulted the large decrease in HIV prevalence observed in these districts, and that this would not have occurred in the absence of Avahan. The syphilis treatment component alone prevented $<9$ and $13 \%$ of new HIV infections over 1 and 10 years. Impact projections for the general population and additional districts will be presented.

Conclusions These Bayesian modelling results, combined with observed HIV prevalence trends and evidence of successful implementation and scale-up of Avahan, provides plausible evidence that Avahan has reduced HIV transmission to a large extent among highrisk groups.

\section{S7.4 COST-EFFECTIVENESS OF TARGETED HIV PREVENTIONS FOR FEMALE SEX WORKERS: AN ECONOMIC EVALUATION OF THE AVAHAN PROGRAMME IN SOUTHERN INDIA}

doi:10.1136/sextrans-2011-050102.30

\begin{abstract}
${ }^{1,2} \mathrm{~S}$ Chandrashkar, ${ }^{1} \mathrm{~A}$ Vassall, ${ }^{1,3} \mathrm{~L}$ Guinness, ${ }^{1,4} \mathrm{M}$ Pickles, ${ }^{5} \mathrm{~B}$ Reddy, ${ }^{5} \mathrm{G}$ Shetty, ${ }^{4} \mathrm{M}$ C Boily, ${ }^{6} \mathrm{~K}$ Lowndes, ${ }^{7,8} \mathrm{M}$ Alary, ${ }^{1} \mathrm{P}$ Vickerman. ${ }^{1}$ London School of Hygiene and Tropical Medicine, London, UK; ${ }^{2}$ St Johns Research Institute, India; ${ }^{3}$ Australian Centre for Economics Research in Health, Australian National University; ${ }^{4}$ Imperial College, London, UK; ${ }^{5}$ Karnataka Health Promotion Trust, Bangalore, India; ${ }^{6}$ Health Protection Agency, London, UK; ${ }^{7}$ URESP, Centre de recherche FRSO du CHA universitaire de Québec, Québec, Canada; ${ }^{8}$ Département de médecine sociale et préventive, Université Laval, Québec, Canada
\end{abstract}

Objective(s) The aim of the presentation paper is to assess the costeffectiveness of HIV prevention interventions for female sex workers in India, in the context of large-scale programme effort, the Avahan Programme.

Design/methods We estimate cost-effectiveness using comprehensive measurements of cost; and, impact estimates based on dynamical transmission models of HIV and STI transmission that are fitted to observed prevalence trends. Our primary outcome measure is incremental cost per DALY averted (ICER) for the HIV prevention programme compared to a "do-nothing" base case.

Results In 2007, the implementing NGO cost per person reached at least once in a year ranges from US\$47 to US\$154. When all costs are taken into account (including expertise enhancement, management and contracting costs), the cost per person reached increases to US\$112 to US\$ 213, depending on location and year. Median incremental costs per infection averted range from US\$ 876 (370, $3040)$ to US $\$ 2574(1344,7132)$. Median incremental costs per DALY averted range from US\$49 (20171) in to US\$ $143(74,388)$. These costs per DALY may increase as results from other states are included.

Conclusions This study presents robust evidence that demonstrates that HIV prevention interventions targeted at high risk vulnerable groups can achieve substantial reductions in HIV infections at an acceptable cost in a concentrated epidemic setting. Moreover, we demonstrate cost-effectiveness in the context of a programme that is delivering HIV prevention at scale. However, the achievement of cost-effectiveness varies by setting. Our findings suggest that those responsible for HIV programmes should therefore consider funding targeted HIV prevention programmes at high risk groups in India and beyond, but should take into account setting specific drivers of the HIV epidemic.

\section{Symposium 8: The global public health chal- lenge of untreatable multidrug-resistant Neis- seria gonorrhoeae "superbug" (MDR-GC) S8.1 LESSONS LEARNT FROM GLOBAL HIV DRUG RESISTANCE}

doi:10.1136/sextrans-2011-050102.31

D Sutherland. Global Public Health, Canada

The WHO, in collaboration with the Bill and Melinda Gates Foundation and the International AIDS Society developed the Global HIV Drug Resistance Network. It is comprised of a network of countries and accredited laboratories. The Network serves as an advisory and evaluation function to the WHO HIV drug resistance team and countries implementing the strategy. Surveillance of HIV drug resistance is critical because it helps to detect the circulation of resistance strains and directs measures to preserve programme effectiveness.

This presentation will explore how lessons learnt from the HIV drug resistance initiative could be applied to slow the spread of MDR-GC.

\section{S8.2 SYNERGY: PUBLIC HEALTH, CLINICIANS, LABORATORIES AND MANAGEMENT GUIDELINES}

doi:10.1136/sextrans-2011-050102.32

I Martin, T Wong. Public Health Agency of Canada, Canada

There is growing concern that the increasing prevalence of AMR in $N$ gonorrhoeae will compromise effective treatment and disease control efforts. Early warning systems and the creation of, public health, clinical and laboratory networks are critical to detect the emergence of resistance and treatment failures.

Using specific examples to illustrate best practises, this presentation will focus on mechanisms to:

Enable adequate, timely AMR surveillance to inform treatment guidelines;

Establish a strategy to rapidly detect patients with gonococcal infections who experience a clinical and/or microbiological treatment failure especially with recommended cephalosporin or azithromycin therapy; and

Promote effective public health and clinical management of patients and their sexual partners.

\section{S8.3 RESEARCH AND TRAINING NEEDS}

doi:10.1136/sextrans-2011-050102.33

\section{J A Dillon. University of Saskatchewan, Canada}

Establishing treatment guidelines, improving capacity to monitor antimicrobial susceptibility, and adequate supply of quality medications are key strategies to slow the spread of resistant gonorrhoea. Advances in the public health and clinical management of 